Pacific Journal of Mathematic 


\title{
TRIDIAGONAL MATRIX REPRESENTATIONS OF CYCLIC SELF-ADJOINT OPERATORS
}

\author{
JOANNE DOMBROWSKI
}

\begin{abstract}
A bounded cyclic self-adjoint operator $C$, defined on a separable Hilbert space, can be represented as a tridiagonal matrix with respect to the basis generated by a cyclic vector. If the main diagonal entries are zeros, $C$ may be regarded as the real part of a weighted shift operator. Define $J$ to be the corresponding imaginary part and it follows that $C J-J C=-2 i K$ where $K$ is a diagonal operator. The main purpose of this paper is to show that if the subdiagonal entries converge to a non-zero limit and if $K$ is of trace class then $C$ has an absolutely continuous part.
\end{abstract}

1. Introduction. Let $C$ be a bounded self-adjoint operator on an infinite dimensional Hilbert space $\mathscr{H}$. If $C$ has a cyclic vector $\phi$ then the Gram-Schmidt orthogonalization process applied to $\left\{\phi, C \phi, C^{2} \phi, \ldots\right\}$ provides a basis $\left\{\phi_{n}\right\}$ for $\mathscr{H}$ which will make the matrix of $C$ tridiagonal:

$$
C=\left[\begin{array}{ccccc}
b_{1} & a_{1} & 0 & 0 & \cdots \\
a_{1} & b_{2} & a_{2} & 0 & \ldots \\
0 & a_{2} & b_{3} & a_{3} & \ldots \\
\vdots & \vdots & \vdots & \vdots &
\end{array}\right], \quad a_{n}>0 .
$$

It is then easily shown (see [4]) that $\phi_{n}=P_{n}(C) \phi_{1}$ with the polynomials $\left\{P_{n}\right\}$ satisfying the following:

$$
\begin{gathered}
P_{1}(\lambda)=1, \quad P_{2}(\lambda)=\frac{\lambda-b_{1}}{a_{1}}, \\
P_{n}(\lambda)=\frac{\left(\lambda-b_{n-1}\right) P_{n-1}(\lambda)-a_{n-2} P_{n-2}(\lambda)}{a_{n-1}}, \quad(n>2) .
\end{gathered}
$$

If $C=\int \lambda d E_{\lambda}$ and $\mu(\beta)=\left\|E(\beta) \phi_{1}\right\|^{2}$ for any Borel set $\beta$, then $C$ can be represented as a multiplication operator on $L^{2}(\mu)$. The polynomials $\left\{P_{n}(\lambda)\right\}$ form an orthonormal basis for this space.

In the following, results on orthogonal polynomials are combined with the techniques of operator theory to study the relation between the constants in the tridiagonal matrix representation of the operator and its spectrum. Throughout it will be assumed that the main diagonal elements in (1.1) are zero. In this case $C$ may be regarded as the real part of the 
shift operator $T$ defined by $T \phi_{n}=2 a_{n} \phi_{n+1}$. Letting $J$ be the corresponding imaginary part it can be shown that $C J-J C=-2 i K$ where $K$ is a diagonal operator with diagonal entries $a_{1}^{2},-a_{1}^{2}+a_{2}^{2},-a_{2}^{2}+a_{3}^{2}, \ldots$ The main purpose of this paper is to study the spectrum of $C$, and, in particular, to establish the existence of an absolutely continuous part, under the assumption that $K$ is of trace class.

Several results in the literature should be noted. In case the sequence $\left\{a_{n}\right\}$ is increasing the operator $C$ is the real part of a hyponormal operator and the existence of an absolutely continuous part follows from results due to Putnam [8]. Commutator techniques are used in [4] to analyze the spectrum if the sequence $\left\{a_{n}\right\}$ decreases monotonically to a non-zero limit. Both of these special cases apply to the study of phase operators which are associated with the phase of the harmonic oscillator. (See [6], [3].) Also, as indicated above, the constants in the matrix representation of $C$ appear in the recursion formula for a system of orthogonal polynomials. Systems of orthogonal polynomials and the corresponding distribution functions (or weight functions) have been studied by Nevai, among others, under various assumptions on the coefficients in the recursion formula. The results obtained by Nevai in [7] identify the absolutely continuous part of $C$ if $\sum\left|a_{n}-\frac{1}{2}\right|<\infty$. Again this condition implies that $K$ is of trace class. These facts motivated the main result to be presented. The remainder of the paper studies the properties of the sequence $\left\{a_{n}\right\}$ under the assumption that the absolutely continuous part of $C$ is trivial.

2. Eigenvalues. It is shown in Stone [10] that $C x=\lambda x$ if and only if $\sum_{n=1}^{\infty}\left|P_{n}(\lambda)\right|^{2}<\infty$. If $\lambda$ is an eigenvalue then $x=\sum x_{n} \phi_{n}$ with $x_{n}=$ $P_{n}(\lambda)$. Also it is shown in [4] that if $C x=\lambda x$ then

$$
\langle K x, x\rangle=a_{1}^{2} P_{1}^{2}(\lambda)+\sum_{n=2}^{\infty}\left(-a_{n-1}^{2}+a_{n}^{2}\right) P_{n}^{2}(\lambda)=0,
$$

and if

$$
S_{N}(\lambda)=a_{1}^{2} P_{1}^{2}(\lambda)+\sum_{n=2}^{N}\left(-a_{n-1}^{2}+a_{n}^{2}\right) P_{n}^{2}(\lambda)
$$

then

$$
S_{N}(\lambda)=\left[a_{N-1} P_{N-1}(\lambda)-\frac{\lambda}{2} P_{N}(\lambda)\right]^{2}+\left(a_{N}^{2}-\frac{\lambda^{2}}{4}\right) P_{N}^{2}(\lambda)
$$


These facts are needed for the first result along with the following notation: $f^{+}(t)=f(t)$ if $f(t) \geq 0$ and $f^{+}(t)=0$ if $f(t)<0 ; f^{-}(t)=|f(t)|$ if $f(t) \leq 0$ and $f^{-}(t)=0$ if $f^{-}(t)>0$.

THEOREM 1. If $\sum_{n=2}^{\infty}\left(-a_{n-1}^{2}+a_{n}^{2}\right)^{-}<\infty$ then $C$ has no eigenvalues in $(-2 \alpha, 2 \alpha)$ where $\alpha=\liminf a_{n}$.

Proof. Assume that $C$ has an eigenvalue $\lambda$ in the interval $(-2 \alpha, 2 \alpha)$. Choose $M$ such that $n>M$ implies that

$$
a_{n}^{2}>\frac{1}{2}\left(\frac{\lambda^{2}}{4}+\alpha^{2}\right) \text { and } \sum_{M+1}^{\infty}\left(-a_{n}^{2}+a_{n}^{2}\right)^{-}<\frac{1}{4}\left(\alpha^{2}-\frac{\lambda^{2}}{4}\right) .
$$

Choose $N$ such that $P_{N}^{2}(\lambda)=\max _{n>M} P_{n}^{2}(\lambda)$. Then for $l>N$

$$
\begin{aligned}
S_{l}(\lambda) \geq & S_{N}(\lambda)-\frac{1}{4}\left(\alpha^{2}-\frac{\lambda^{2}}{4}\right) P_{N}^{2}(\lambda) \\
= & {\left[a_{N-1} P_{N-1}(\lambda)-\frac{\lambda}{2} P_{N}(\lambda)\right]^{2}+\left(a_{N}^{2}-\frac{\lambda^{2}}{4}\right) P_{N}^{2}(\lambda) } \\
& -\frac{1}{4}\left(\alpha^{2}-\frac{\lambda^{2}}{4}\right) P_{N}^{2}(\lambda) \\
\geq & \frac{1}{4}\left(\alpha^{2}-\frac{\lambda^{2}}{4}\right) P_{N}^{2}(\lambda)
\end{aligned}
$$

If $P_{N}(\lambda) \neq 0$ then $\lambda$ cannot be an eigenvalue. The same is true if $P_{N}(\lambda)=0$ and $S_{N}(\lambda) \neq 0$. If $P_{N}(\lambda)=0$ and $S_{N}(\lambda)=0$ then $P_{N-1}(\lambda)=$ 0 and the recursion formula (1.2) implies that $P_{n}(\lambda)=0$ for $1 \leq n \leq N-$ 1 which contradicts the fact that $P_{1}(\lambda)=1$.

3. Absolute continuity. If $C=\int \lambda d E_{\lambda}$, let $\mathscr{H}_{\mathrm{ac}}(C)$ denote the set of elements $x$ in $\mathscr{H}$ for which $\left\|E_{\lambda} x\right\|^{2}$ is an absolutely continuous function of $\lambda$. It is known that $\mathscr{H}_{\mathrm{ac}}(C)$ is a subspace that reduces $C$. The restriction of $C$ to this subspace is called the absolutely continuous part of $C$. The following lemma, due to Putnam [8, see the proof of Theorem 2.2.4], is needed for the next result.

Lemma. If $C$ and $J$ are bounded self-adjoint operators defined on $\mathscr{H}$ and if $C J-J C=2 i K$ then for any interval $\Delta$ and any $x$ in $\mathscr{H}$,

$$
|\langle K E(\Delta) x, E(\Delta) x\rangle| \leq\|J\||\Delta|\|E(\Delta) x\|^{2},
$$

where $|\Delta|$ denotes the length of $\Delta$. 
It will now be assumed that $\lim a_{n}=\frac{1}{2}$. Since it is well known that the spectrum of the real part of the unilateral shift operator is exactly the interval $[-1,1]$, Weyl's Theorem guarantees that the spectrum of $C$ contains the interval $[-1,1]$ and that any points in the spectrum outside of this interval must be eigenvalues.

THEOREM 2. If $\lim a_{n}=\frac{1}{2}$ and if $\sum\left|-a_{n-1}^{2}+a_{n}^{2}\right|<\infty$ then $C$ has a non-trivial absolutely continuous part whose spectrum contains the interval $(-1,1)$.

Proof. Given $k>1$ choose $M$ such that $n>M$ implies that

$$
\left|a_{n}^{2}-\frac{1}{4}\right|<\frac{1}{16 k} \text { and } \sum_{M+1}^{\infty}\left|-a_{n-1}^{2}+a_{n}^{2}\right|<\frac{1}{8 k} \text {. }
$$

Consider the operator $C_{k}$ whose first $M$ weights are $\frac{1}{2}$, with the remaining weights equal to those of $C$. It will first be shown that the spectrum of the absolutely continuous part of $C_{k}$ contains the interval $(-1+1 / k, 1-$ $1 / k)$. Toward this end let $\Delta$ be a subinterval of $(-1+1 / k, 1-1 / k)$. Several cases need to be considered. Let $\mu(\Delta)=\left\|E(\Delta) \phi_{1}\right\|^{2}$, and note that

$\left\langle K E(\Delta) \phi_{1}, E(\Delta) \phi_{1}\right\rangle=a_{1}^{2}\left|\int_{\Delta} P_{1} d \mu\right|^{2}+\sum_{n=2}^{\infty}\left(-a_{n-1}^{2}+a_{n}^{2}\right)\left|\int_{\Delta} P_{n} d \mu\right|^{2}$.

Case I. Suppose $\int_{\Delta} P_{n}^{2} d \mu \leq \mu(\Delta)=\int_{\Delta} P_{1}^{2} d \mu$ for each $n$. Then

$$
\begin{aligned}
\left|\left\langle K E(\Delta) \phi_{1}, E(\Delta) \phi_{1}\right\rangle\right| & \geq a_{1}^{2}|\mu(\Delta)|^{2}-\mu(\Delta) \sum\left|-a_{n-1}^{2}+a_{n}^{2}\right| \int_{\Delta} P_{n}^{2} d \mu . \\
& \geq|\mu(\Delta)|^{2} \frac{1}{4}\left(1-\frac{1}{2 k}\right) .
\end{aligned}
$$

Case II. Suppose $\int_{\Delta} P_{n}^{2} d \mu \leq \mu(\Delta)$ except for a finite number of $n$. Then there exists $N$ such that $\int_{\Delta} P_{N}^{2} d \mu=\max _{n} \int_{\Delta} P_{n}^{2} d \mu$. It follows that 


$$
\begin{aligned}
\left|\left\langle K E(\Delta) \phi_{1}, E(\Delta) \phi_{1}\right\rangle\right| \geq & a_{1}^{2}\left|\int_{\Delta} d \mu\right|^{2}+\mu(\Delta) \sum_{n=2}^{N}\left(-a_{n-1}^{2}+a_{n}^{2}\right) \int_{\Delta} P_{n}^{2} d \mu \\
& -\mu(\Delta) \sum_{n=2}^{N}\left(-a_{n-1}^{2}+a_{n}^{2}\right)^{+} \int_{\Delta} P_{n}^{2} d \mu \\
& -\sum_{N+1}^{\infty}\left|-a_{n-1}^{2}+a_{n}^{2}\right|\left|\int_{\Delta} P_{n} d \mu\right|^{2} .
\end{aligned}
$$

It then follows from (2.2) that

$$
\begin{aligned}
\left|\left\langle K E(\Delta) \phi_{1}, E(\Delta) \phi_{1}\right\rangle\right| \\
\geq \mu(\Delta)\left[\int_{\Delta}\left[a_{N-1} P_{N-1}-\frac{\lambda}{2} P_{N}\right]^{2} d \mu+\int_{\Delta}\left(a_{N}^{2}-\frac{\lambda^{2}}{4}\right) P_{N}^{2} d \mu\right] \\
\quad-\frac{1}{8 k} \mu(\Delta) \int_{\Delta} P_{N}^{2} d \mu \\
\geq \frac{1}{16 k}|\mu(\Delta)|^{2} .
\end{aligned}
$$

Case III. Suppose there exists a subsequence $\left\{P_{n_{j}}\right\}$ such that $\int_{\Delta} P_{n_{j}}^{2} d \mu$ $\geq \mu(\Delta)$. Choose $L$ sufficiently large such that

$$
\begin{aligned}
\sum_{L+1}^{\infty}\left|-a_{n-1}^{2}+a_{n}^{2}\right|\left|\int_{\Delta} P_{n} d \mu\right|^{2} & \leq \mu(\Delta) \sum_{L+1}^{\infty}\left|-a_{n-1}^{2}+a_{n}^{2}\right| \\
& \leq \frac{1}{32 k}|\mu(\Delta)|^{2}
\end{aligned}
$$

Choose $P_{M}$ such that $M>L$ and $\int_{\Delta} P_{M}^{2} d \mu \geq \mu(\Delta)$. Choose $P_{N}$ such that $\int_{\Delta} P_{N}^{2} d \mu=\max _{1 \leq n \leq M} \int_{\Delta} P_{n}^{2} d \mu$. Then

$\left|\left\langle K E(\Delta) \phi_{1}, E(\Delta) \phi_{1}\right\rangle\right|$

$$
\begin{aligned}
\geq & a_{1}^{2}\left|\int_{\Delta} P_{1} d \mu\right|^{2}+\mu(\Delta) \sum_{n=2}^{N}\left(-a_{n-1}^{2}+a_{n}^{2}\right) \int_{\Delta} P_{n}^{2} d \mu \\
& -\mu(\Delta) \sum_{n=2}^{N}\left(-a_{n-1}^{2}+a_{n}^{2}\right)^{+} \int_{\Delta} P_{n}^{2} d \mu \\
& -\sum_{N+1}^{M}\left|-a_{n-1}^{2}+a_{n}^{2}\right|\left|\int_{\Delta} P_{n} d \mu\right|^{2}-\sum_{M+1}^{\infty}\left|-a_{n-1}^{2}+a_{n}^{2}\right|\left|\int_{\Delta} P_{n} d \mu\right|^{2} .
\end{aligned}
$$


Hence

$$
\begin{aligned}
&\left|\left\langle K E(\Delta) \phi_{1}, E(\Delta) \phi_{1}\right\rangle\right| \\
& \geq {\left[\int_{\Delta}\left(a_{N-1} P_{N-1}-\frac{\lambda}{2} P_{N}\right)^{2} d \mu+\int_{\Delta}\left(a_{N}^{2}-\frac{\lambda^{2}}{4}\right) P_{N}^{2} d \mu\right] \mu(\Delta) } \\
&-\frac{1}{8 k} \mu(\Delta) \int_{\Delta} P_{N}^{2} d \mu-\frac{1}{32 k}|\mu(\Delta)|^{2} \\
& \geq \frac{1}{32 k}|\mu(\Delta)|^{2} .
\end{aligned}
$$

In any case, therefore,

$$
\left|\left\langle K E(\Delta) \phi_{1}, E(\Delta) \phi_{1}\right\rangle\right| \geq \frac{1}{32 k}|\mu(\Delta)|^{2} .
$$

This result and the previous lemma imply that $|\mu(\Delta)| \leq 32 k\|J\||\Delta|$. If $\beta$ is a Borel subet of $(-1+1 / k, 1-1 / k)$ of Lebesgue measure zero, then for any $\varepsilon>0$ there exists a pairwise disjoint sequence of intervals $\left\{\Delta_{j}\right\}$ such that $\Delta_{j} \subset(-1+1 / k, 1-1 / k), \beta \subset U \Delta_{j}$ and $\Sigma\left|\Delta_{j}\right|<\varepsilon$. Then $\mu(\beta) \leq$ $\Sigma_{j} \mu\left(\Delta_{j}\right) \leq 32 k\|J\| \Sigma\left|\Delta_{j}\right|$ and it readily follows that $\mu(\beta)=0$. Hence $C_{k}$ has a non-trivial absolutely continuous part whose spectrum contains the interval $(-1+1 / k, 1-1 / k)$. Recall that $C$ and $C_{k}$ differ only in a finite number of weights. Thus $C$ is a trace class perturbation of $C_{k}$ and it follows from the Kato-Rosenblum Theorem that the spectrum of the absolutely continuous part of $C$ must contain $(-1+1 / k, 1-1 / k)$ for each $k$ as was to be shown.

4. Singular operators. It was shown in [2] that the condition $\liminf a_{n}>0$ is necessary for the existence of a non-trivial absolutely continuous part for $C$. Assume now that $C$ is a cyclic self-adjoint operator for which $\mathscr{H}_{\text {ac }}(C)$ is trivial. Must it follow that $\lim \inf a_{n}=0$ ? It will be shown below that the problem can be simplified. Sufficient conditions will then be presented for an affirmative answer. Throughout it will be assumed that $\|C\|=1$ so that $\operatorname{sp}(C) \subset[-1,1]$.

For any self-adjoint operator $C$ the orthogonal complement of $\mathscr{H}_{\mathrm{ac}}(C)$ can be decomposed as $\mathscr{H}_{p}(C) \oplus \mathscr{H}_{\mathrm{sc}}(C)$ with $\mathscr{H}_{p}(C)$ generated by the eigenvectors of $C$. In [1] Cary and Pincus have shown that if $\mathscr{H}_{\mathrm{sc}}(C)=\mathscr{H}$ then there exists a cyclic vector $y$ such that $C+y \otimes y$ is diagonal. It is easily seen that the matrix of $C$ with respect to the basis obtained by orthonormalizing $\left\{y, C y, C^{2} y, \ldots\right\}$ differs from the matrix $D=C+y \otimes y$ with respect to the same basis only in the first diagonal element. It is reasonable, therefore, to consider cyclic self-adjoint diagonal operators. Several results follow. 
In case $C$ is compact, so that the spectrum of $C$ has exactly one limit point, it is easily shown that $\lim a_{n}=0$.

THEOREM 3. If the spectrum of $C$ is symmetric about the origin with exactly two limit points then $\lim \inf a_{n}=0$. In particular, if $C$ is invertible then $\lim a_{2 k}=0$ and if $C$ is not invertible then $\lim a_{2 k+1}=0$.

Proof. Assume, without loss of generality, that 1 and -1 are the limit points of the spectrum. From the matrix representation $a_{n}=\int \lambda P_{n} P_{n+1} d \mu$. Since $P_{2 k} / \lambda$ is a polynomial of degree $(2 k-2), \int P_{2 k} / \lambda P_{2 k+1} d \mu=0$. Hence $a_{2 k}=\int\left(\lambda^{2}-1\right) P_{2 k} / \lambda P_{2 k+1} d \mu$. If the spectrum of $C$, which is the support of the measure $\mu$, is bounded away from the origin then Holders Inequality and the fact that the sequence $\left\{P_{k}\right\}$ converges pointwise to zero on the spectrum of $C$ imply that $\lim a_{2 k}=0$.

Now assume that $C$ is not invertible. The recursion formula and an induction argument show that $a_{n}^{2}+a_{n+1}^{2}=\int \lambda^{2} P_{n+1}^{2} d \mu$. Hence

$$
\begin{aligned}
a_{2 k}^{2} & =\int \lambda^{2}\left(P_{2 k}^{2}-P_{2 k-1}^{2}+P_{2 k-2}^{2}-\cdots\right) d \mu \\
& =\int\left(\lambda^{2}-1\right)\left(P_{2 k}^{2}-P_{2 k-1}^{2}+P_{2 k-2}^{2}-\cdots\right) d \mu .
\end{aligned}
$$

Let $X(\lambda)$ be the characteristic function of the singleton set $\{0\}$. Then

$$
\mu(\{0\})=\int X^{2} d \mu=\sum_{n=1}^{\infty}\left|\int X(\lambda) P_{n}(\lambda) d \mu\right|^{2}=\sum_{n=1}^{\infty}\left|P_{n}(0) \mu(\{0\})\right|^{2} .
$$

If $\mu(\{0\}) \neq 0$ then $\sum_{n=1}^{\infty} P_{n}^{2}(0)=1 / \mu(\{0\})$. Since $P_{2 k}(0)=0$ for $k=$ $1,2, \ldots$, it follows that

$$
a_{2 k}^{2}=1+\int_{\lambda \neq 0}\left(\lambda^{2}-1\right)\left(P_{2 k}^{2}-P_{2 k-1}^{2}+P_{2 k-2}^{2}-\cdots\right) d \mu .
$$

Using the Christoffel-Darboux summation formula [11, Theorem 3.2.2]

$$
\sum_{k=1}^{n} P_{k}(\xi) P_{k}(\lambda)=a_{n} \frac{P_{n}(\xi) P_{n+1}(\lambda)-P_{n+1}(\xi) P_{n}(\lambda)}{\lambda-\xi}
$$

with $\xi=-\lambda$, it can be shown that the integral on the right converges to zero. Hence $\lim _{k \rightarrow \infty} a_{2 k}^{2}=1$ and since $\lim _{k \rightarrow \infty}\left(a_{2 k}^{2}+a_{2 k+1}^{2}\right)=1$ it follows that $\lim a_{2 k+1}^{2}=0$ as was to be shown.

THEOREM 4. If the spectrum of $C$ has a finite number of limit points then $\liminf a_{n}=0$. 
Proof. Suppose $\lambda_{1}, \lambda_{2}, \ldots, \lambda_{k}$ are the limit points of the spectrum of $C$. Let $\pi(\lambda)=\left(\lambda-\lambda_{1}\right)\left(\lambda-\lambda_{2}\right) \cdots\left(\lambda-\lambda_{k}\right)$. Then $\pi(\lambda) P_{n}(\lambda)$ can be expressed as a linear combination of $P_{1}, P_{2}, \ldots, P_{n+k}$ with the coefficient of $P_{n+k}$ equal to $a_{n} a_{n+1} \cdots a_{n+k-1}$. Hence $\int \pi(\lambda) P_{n} P_{n+k} d \mu=a_{n} \cdots$ $a_{n+k-1}$. Let $\left\{\xi_{l}\right\}$ be the support of the measure $\mu$. Then given $\varepsilon>0$ there exists $M$ such that for $i>M,\left|\pi\left(\xi_{l}\right)\right|<\varepsilon / 2$. Also there exists $N$ such that for $n>N,\left|\int_{E} \pi(\lambda) P_{n} P_{n+k} d \mu\right|<\varepsilon / 2$ where $E=\left\{\xi_{1}, \xi_{2}, \ldots, \xi_{M}\right\}$. For $n$ $>N,\left|\int \pi(\lambda) P_{n} P_{n+k} d \mu\right|=a_{n} a_{n+1} \cdots a_{n+k-1}<\varepsilon$. Thus the sequence $\left\{a_{n} a_{n+1} \cdots a_{n+k-1}\right\}$ converges to zero and hence $\liminf a_{n}=0$ as was to be shown.

THEOREM 5. If for each $n$ there exists a set of $2 n$ points in $[-1,1]$ such that all but a finite number of the atoms of $\mu$ are within $\varepsilon_{n}$ of this set, with $\lim \left(\varepsilon_{n}\right)^{1 / n}=0$, then $\liminf a_{n}=0$.

Proof. Fix $n$ and let $M=\left\{\xi_{1}, \xi_{2}, \ldots, \xi_{2 n}\right\}$ be the corresponding set. If $A$ denotes the set of atoms within $\varepsilon_{n}$ of $M$ and if $B$ denotes the remaining finite set of atoms, then for any $k$,

$$
\begin{aligned}
& \left(a_{k} a_{k+1} \cdots a_{k+2 n-1}\right)^{1 / 2 n} \\
& =\left[\int_{A}\left(\lambda-\xi_{1}\right) \cdots\left(\lambda-\xi_{2 n}\right) P_{k}(\lambda) P_{k+2 n}(\lambda) d \mu\right. \\
& \left.\quad \quad \quad \int_{B}\left(\lambda-\xi_{1}\right) \cdots\left(\lambda-\xi_{2 n}\right) P_{k}(\lambda) P_{k+2 n}^{(\lambda)} d \mu\right]^{1 / 2 n} .
\end{aligned}
$$

Choose $k$ sufficiently large so that $\left|P_{k}(\lambda)\right|<\varepsilon_{n}$ for any $\lambda$ in $B$. It then follows from Holder's Inequality that

$$
\left(a_{k} a_{k+1} \cdots a_{k+2 n-1}\right)^{1 / 2 n} \leq\left(2^{2 n} \cdot \varepsilon_{n}+2^{2 n} \cdot \varepsilon_{n}\right)^{1 / 2 n} \leq 4 \varepsilon_{n}^{1 / 2 n}
$$

which implies that $\liminf a_{n}=0$.

Theorem 5 can be used to construct an example of a diagonal operator whose spectrum has an infinite number of limit points and yet $\liminf a_{n}=0$.

EXAmple. The aim is to define an atomic measure $\mu$ on $[-1,1]$ so that the limit points of the support of the measure are $1,-1,1-1 / 2^{n^{2}}$, $-1+1 / 2^{n^{2}}(n=1,2, \ldots)$. To this end define the positive atoms by the following scheme:

$$
\frac{1}{2}+\frac{1}{4} ; \frac{1}{2}+\frac{1}{8}, 1-\frac{1}{2^{4}}+\frac{1}{16} ; \frac{1}{2}+\frac{1}{32}, 1-\frac{1}{2^{4}}+\frac{1}{64}, 1-\frac{1}{2^{9}}+\frac{1}{128} ;
$$


etc. Define the negative atoms symmetrically and assign weights to these points so that $\mu[-1,1]=1$. If $C f(\lambda)=\lambda f(\lambda)$ for $f$ in $L^{2}(\mu)$ then $C$ has a tridiagonal matrix representation with respect to the basis $\left\{P_{n}(\lambda)\right\}$ and by Theorem $5 \lim \inf a_{n}=0$. (Note that the conditions of the Theorem are satisfied with $\varepsilon_{n}=1 / 2^{n^{2}}$.)

The previous results depend on the placement of the atoms of the discrete measure $\mu$. The final result of this section depends on the rate of convergence of the measure on the sequence of eigenvalues.

THEOREM 6. If $\left\{\lambda_{k}\right\}$ is the set of eigenvalues for the cyclic diagonal operator $C$ and if $\left[\sum_{k>n} \mu\left(\lambda_{k}\right)\right]^{1 / 2 n}$ converges to zero then $\lim \inf a_{n}=0$.

Proof. Let $A_{n}=\bigcup_{k>n}\left\{\lambda_{k}\right\}$. Then

$$
\begin{aligned}
\left(a_{1} a_{2} \cdots a_{n}\right)^{1 / n} & =\left(\int_{A_{n}}\left(\lambda-\lambda_{1}\right) \cdots\left(\lambda-\lambda_{n}\right) P_{n+1} d \mu\right)^{1 / n} \\
& \leq 2\left(\int_{A_{n}} P_{n+1}^{2} d \mu\right)^{1 / 2 n}\left(\int_{A_{n}} d \mu\right)^{1 / 2 n} \leq 2\left[\sum_{k>n} \mu\left(\lambda_{k}\right)\right]^{1 / 2 n} .
\end{aligned}
$$

It follows that $\liminf a_{n}=0$.

REMARK. It is of course possible to define an atomic measure on $[-1,1]$ so that the atoms are dense in $[-1,1]$ and the convergence condition of Theorem 6 fails. It would be interesting to know $\lim \inf a_{n}$ under these conditions.

\section{REFERENCES}

[1] R. W. Carey and J. D. Pincus, Unitary equivalence modulo the trace class for self-adjoint operators, Amer. J. Math., 98 (1976), 481-514.

[2] J.Dombrowski, Quasitriangular matrices, Proc. Amer. Math. Soc., 69 (1978), 95-96.

[3] __ Spectral properties of phase operators, J. Math. Phys., 15 (1974), 576-577.

[4] Spectral properties of real parts of weighted shift operators, Indiana Univ. Math. J., 29 (1980), 249-259.

[5] T. Kato, Perturbation of continuous spectra by trace class operators, Proc. Japan Acad., 33 (1957), 260-264.

[6] E. C. Lerner, H. W. Huang and G. E. Walters, Some mathematical properties of oscillator phase operators, J. Math. Phys., 11 (1970), 1979-1984.

[7] P. G. Nevai, Orthogonal Polynomials, Mem. Amer. Math. Soc., Vol. 18, 1979.

[8] C. R. Putnam, Commutation Properties of Hilbert Space Operators and Related Topics, Ergebnesse der Math., 36, Springer, 1967.

[9] M. Rosenblum, Perturbation of the continuous spectrum and unitary equivalence, Pacific J. Math., 7 (1957), 997-1010. 
[10] M. H. Stone, Linear Transformations in Hilbert Space, Amer. Math. Soc., New York, 1932.

[11] Szego, Orthogonal Polynomials, Amer. Math. Soc., Providence, R. I., 1939, Fourth edition, 1975.

Received November 17, 1982.

WRIGHT State UNIVERSITY

DAYTON, OH 45435 


\section{PACIFIC JOURNAL OF MATHEMATICS EDITORS}

\author{
DONALD BABBITT (Managing Editor) \\ University of California \\ Los Angeles, CA 90024 \\ J. DugundJI \\ University of Southern California \\ Los Angeles, CA 90089-1113 \\ R. FINN \\ Stanford University \\ Stanford, CA 94305 \\ HERMANN FLASChKa \\ University of Arizona \\ Tucson, AZ 85721
}

C. C. MOORE

University of California

Berkeley, CA 94720

Arthur Ogus

University of California

Berkeley, CA 94720

Hugo RossI

University of Utah

Salt Lake City, UT 84112

H. SAMELSON

Stanford University

Stanford, CA 94305

ASSOCIATE EDITORS
R. ARENS
E. F. BECKENBACH
B. H. NeUmanN
F. WOLF
K. YosHIDA (1906-1982)

\section{SUPPORTING INSTITUTIONS}
UNIVERSITY OF ARIZONA
UNIVERSITY OF BRITISH COLUMBIA
CALIFORNIA INSTITUTE OF TECHNOLOGY
UNIVERSITY OF CALIFORNIA
MONTANA STATE UNIVERSITY
UNIVERSITY OF NEVADA, RENO
NEW MEXICO STATE UNIVERSITY
OREGON STATE UNIVERSITY

\author{
UNIVERSITY OF OREGON \\ UNIVERSITY OF SOUTHERN CALIFORNIA \\ STANFORD UNIVERSITY \\ UNIVERSITY OF HAWAII \\ UNIVERSITY OF TOKYO \\ UNIVERSITY OF UTAH \\ WASHINGTON STATE UNIVERSITY \\ UNIVERSITY OF WASHINGTON
}

The Supporting Institutions listed above contribute to the cost of publication of this Journal, but they are not owners or publishers and have no responsibility for its content or policies.

Mathematical papers intended for publication in the Pacific Journal of Mathematics should be in typed form or offset-reproduced (not dittoed), double spaced with large margins. Please do not use built up fractions in the text of the manuscript. However, you may use them in the displayed equations. Underline Greek letters in red, German in green, and script in blue. The first paragraph must be capable of being used separately as a synopsis of the entire paper. In particular it should contain no bibliographic references. Please propose a heading for the odd numbered pages of less than 35 characters. Manuscripts, in triplicate, may be sent to any one of the editors. Please classify according to the scheme of Math. Reviews, Index to Vol. 39. Supply name and address of author to whom proofs should be sent. All other communications should be addressed to the managing editor, or Elaine Barth, University of California, Los Angeles, California 90024.

There are page-charges associated with articles appearing in the Pacific Journal of Mathematics. These charges are expected to be paid by the author's University, Government Agency or Company. If the author or authors do not have access to such Institutional support these charges are waived. Single authors will receive 50 free reprints; joint authors will receive a total of 100 free reprints. Additional copies may be obtained at cost in multiples of 50 .

The Pacific Journal of Mathematics is issued monthly as of January 1966. Regular subscription rate: $\$ 190.00$ a year (5 Vols., 10 issues). Special rate: $\$ 66.00$ a year to individual members of supporting institutions.

Subscriptions, orders for numbers issued in the last three calendar years, and changes of address should be sent to Pacific Journal of Mathematics, P.O. Box 969, Carmel Valley, CA 93924, U.S.A. Old back numbers obtainable from Kraus Periodicals Co., Route 100, Millwood, NY 10546.

The Pacific Journal of Mathematics at P.O. Box 969, Carmel Valley, CA 93924 (ISSN 0030-8730) publishes 5 volumes per year. Application to mail at Second-class postage rates is pending at Carmel Valley, California, and additional mailing offices. Postmaster: Send address changes to Pacific Journal of Mathematics, P.O. Box 969, Carmel Valley, CA 93924.

PUBLISHED BY PACIFIC JOURNAL OF MATHEMATICS, A NON-PROFIT CORPORATION

Copyright $\odot 1984$ by Pacific Journal of Mathematics 


\section{Pacific Journal of Mathematics}

Vol. 114, No. $2 \quad$ June, 1984

William Allen Adkins, A Harnack estimate for real normal surface

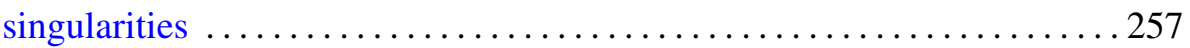

George E. Andrews, Multiple series Rogers-Ramanujan type identities . . . . 267

Didier Arnal, $*$ products and representations of nilpotent groups . . . . . . 285

David Cox and Walter Raymond Parry, Representations associated with

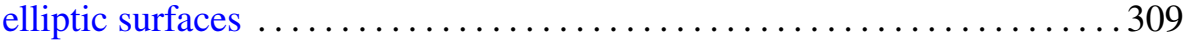

Joanne Marie Dombrowski, Tridiagonal matrix representations of cyclic

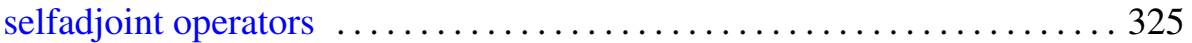

Ronald Dotzel, An Artin relation $(\bmod 2)$ for finite group actions on

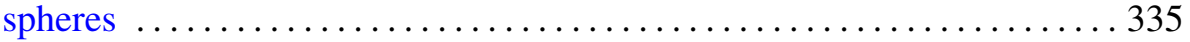

Leo Egghe, Convergence of adapted sequences of Pettis-integrable

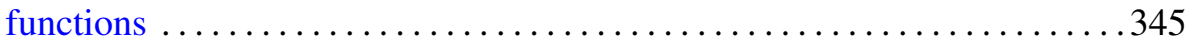

Rebecca A. Herb, Characters of induced representations and weighted

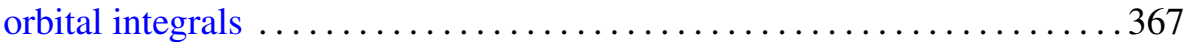

Steven M. Kahn, Cobordism obstructions to fibering manifolds over

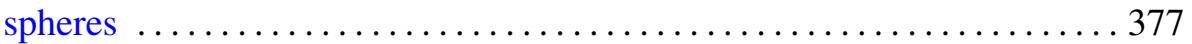

Robert D. Little, Projective space as a branched covering of the sphere with orientable branch set

Claude Schochet, Topological methods for $C^{*}$-algebras. III. Axiomatic

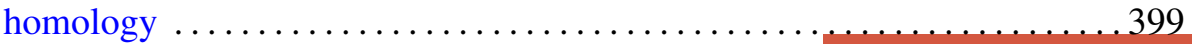

Claude Schochet, Topological methods for $C^{*}$-algebras. IV. $\bmod p$ homology

James M. Stormes, On the $K O$-orientability of complex projective varieties

Josephine Anne Ward, Characterization of homogeneous spaces and their norms 